\title{
AXIOMATIC FOUNDATIONS OF NONRELATIVISTIC QUANTUM MECHANICS: A REALISTIC APPROACH ${ }^{\dagger}$
}

\author{
S. E. Perez Bergliaffa* and H. Vucetich* \\ Departamento de Física, UNLP, C.C. 67, La Plata, C.P. 1900, Argentina \\ and \\ G. E. Romero* \\ Instituto Argentino de Radioastronomía, C.C. 5, Villa Elisa, C.P. 1894, \\ Argentina
}

December, 1993

\begin{abstract}
A realistic axiomatic formulation of nonrelativistic quantum mechanics for a single microsystem with spin is presented, from which the most important theorems of the theory can be deduced. In comparison with previous formulations, the formal aspect has been improved by the use of certain mathematical theories, such as the theory of equipped spaces, and group theory. The standard formalism is naturally obtained from the latter, starting from a central primitive concept: the Galilei group.
\end{abstract}

${ }^{\dagger}$ Published in International Journal of Theoretical Physics, 32, 9, 1993.

* CONICET

E-mail: bergliaffa@fisilp.edu.ar 


\section{INTRODUCTION}

Every physical theory is a hypothetical-deductive system. This system can be presented in several different ways. In our opinion, the axiomatic approach has plenty of advantages when compared with others. Firstly, in an axiomatization, all the presuppositions of the theory are explicited. This is very important to clarify the foundations of the theory. Secondly, there is no place for doubts about the arguments of the functions that appear in the statements. In this way, possible erroneous identification of the physical referents can be avoided. Thirdly, the meanings are assigned by semantical axioms, and not by context. This excludes the frequent mistakes originating in an abuse of analogy. Finally, the axiomatic formulation paves the way to the deduction of new theorems and the elimination of pseudotheorems, because it clarifies the structure of the theory.

The proliferation of interpretations in the case of Quantum Mechanics (QM) is partially due to semantical confusions arising from the non-explicitation of the presuppositions. The standard axiomatization of QM Von Neumann, 1955 has semantical contradictions, because it contains predicates that are not related to the primitives that constitute the basis of the theory [Bunge, 1967a], Bunge, 1973]. Bunge has carried out a realistic axiomatization of QM, from which it is possible to deduce the standard theorems of the nonrelativistic theory Bunge, 1967a], Bunge, 1967b . The present work is a reformulation of that axiomatization which includes several improvements. More specifically:

1. Our axiomatization has been formulated in an abstract way, in the sense that it does not depend on any particular representation, and presents the Schrödinger equation, the Heisenberg equation, and the hamiltonian of a free microsystem as theorems.

2. The use of group theory enhaces the role played by symmetries in QM.

3. The mass and the charge have been eliminated from the generating basis (which has in consequence been reduced). Both properties are introduced by means of operators.

4. The spin is brought out directly from the rotational symmetry of the system.

5. The theory of generalized functions developed by Gel'fand and Shilov enables us to treat all the operators on an equal footing by the use of the equipped Hilbert space.

6. Bargmann's superselection rules are presented as theorems.

In spite of the formal changes, the reference class of the theory remains the same. This implies that the interpretation of our formulation is realistic and literal.

In the first section of this work, we describe some tools to be used in the axiomatization. In the second section we give our axiomatic formulation: background, definitions, axioms and theorems. In the third section, we briefly discuss some semantical aspects, and finally, in the last section, the conclusions.

\section{TOOLS}

We give next some mathematical and physical concepts that will be used in the axiomatic core of the theory. 


\subsection{THE GALILEI GROUP}

The proper Galilei group Bargmann, 1954, Lèvy-Leblond, 1963 contains the temporal and spatial translations, the pure Galilei transformations, and the spatial rotations. A general element of the group has the form

$$
g=(\tau, \vec{a}, \vec{v}, R)
$$

where $\tau$ is a real number, $\vec{a}$ and $\vec{v}$ are arbitrary vectors, and $R$ is an orthogonal transformation. If $\vec{x}$ is a position vector and $t$ is the time, a transformation belonging to the Galilei group is

$$
\begin{aligned}
\overrightarrow{x^{\prime}} & =R \vec{x}+\vec{v} t+\vec{a} \\
t^{\prime} & =t+\tau
\end{aligned}
$$

The multiplication law is given by

$$
\begin{aligned}
g_{1} g_{2} & =\left(\tau_{1}, \overrightarrow{a_{1}}, \overrightarrow{v_{1}}, R_{1}\right)\left(\tau_{2}, \overrightarrow{a_{2}}, \overrightarrow{v_{2}}, R_{2}\right) \\
& =\left(\tau_{1}+\tau_{2}, \overrightarrow{a_{1}}+R_{1} \overrightarrow{a_{2}}+\tau_{2} \overrightarrow{v_{1}}, \overrightarrow{v_{1}}+R_{1} \overrightarrow{v_{2}}, R_{1} R_{2}\right)
\end{aligned}
$$

The unit element of the group is

$$
e=(0,0,0,1)
$$

and the inverse element of $g$ is

$$
g^{-1}=\left(-\tau,-R^{-1}(\vec{a}-\tau \vec{v}),-R^{-1} \vec{v}, R^{-1}\right)
$$

Inönu and Wigner Inönu, 1952 have shown that the basis functions of the representations of the Galilei group cannot be interpreted as wave functions of physical microsystems, because it is impossible to construct well-localized states or states with a definite velocity with them. Moreover, Hamermesh Hamermesh, 1960 pointed out that the position operator can only be constructed in the case of nontrivial ray representations.

Bargmann [Bargmann, 1954] has shown that the physical representations of the Galilei group are obtained from the unitary ray representations of the universal covering group of the Galilei group. The exponents of these physical representations have the form

$$
\xi\left(\overline{g_{1}}, \overline{g_{2}}\right)=\frac{1}{2}\left\{\overrightarrow{a_{1}} \cdot R_{1} \overrightarrow{v_{2}}-\overrightarrow{v_{1}} \cdot R_{1} \overrightarrow{a_{2}}+\tau_{2} \overrightarrow{v_{1}} \cdot R_{1} \overrightarrow{v_{2}}\right\}
$$

where $\overline{g_{1}}=\left(\tau_{1}, \overrightarrow{a_{1}}, \overrightarrow{v_{1}}, R_{1}\right), \overline{g_{2}}=\left(\tau_{2}, \overrightarrow{a_{2}}, \overrightarrow{v_{2}}, R_{2}\right)$ are elements of the universal covering group. To these elements correspond the unitary operators $\hat{U}\left(\overline{g_{1}}\right)$ and $\hat{U}\left(\overline{g_{2}}\right)$ such that

$$
\hat{U}\left(\overline{g_{1}}\right) \hat{U}\left(\overline{g_{2}}\right)=e^{i \xi\left(\overline{g_{1}}, \overline{g_{2}}\right)} \hat{U}\left(\overline{g_{1}}, \overline{g_{2}}\right)
$$

It is possible to construct a local group $\tilde{G}$ in the form

$$
\tilde{G}=(\theta, \bar{G})
$$

where $\theta \in \mathcal{R}$ and $\bar{G}$ is the universal covering group of the Galilei group $G$. We say that $\tilde{G}$ is a nontrivial central extension of the universal covering group $\bar{G}$ of the Galilei group $G$ by a one-dimensional abelian group.

The structure of $\bar{G}$ is locally determined by the structure of its Lie algebra. The commutation relations among the elements of the basis of the algebra can be calculated from the composition laws of $G$. For the generators of spatial translations $\left(\hat{P}_{i}\right)[\mathbf{1}]$ and the generators of pure Galilei transformations $\left(\hat{K}_{i}\right)$, the commutator is identically zero; if we compute this commutator for the elements of the physical representation Lèvy-Leblond, 1963 we get $\left[\hat{K}_{i}, \hat{P}_{j}\right]=\hat{M} \delta_{i j}$. We carry out the central extension of $\bar{G}$ imposing this last 
relation, in such a way that $\hat{M}$ is the element of the Lie algebra of the one-parameter subgroup used in the extension. This extension is central because $\hat{M}$ commutes with all the other elements of the algebra, and it is nontrivial because $\hat{M}$ appears on the right side of some commutation relations. The physical representations are then the representations of the algebra of the central extension of $\bar{G}$.

In our axiomatic formulation, the commutation relations of the algebra of $\tilde{G}$ are explicitly postulated, and the generator of the algebra of the one-parameter subgroup is identificated with the mass operator $\hat{M}$.

Let's turn now to the equipped Hilbert spaces and Gel'fand's theorem.

\subsection{EQUIPPED HILBERT SPACES}

As is well known, not all the physically important operators appearing in QM have eigenfunctions with finite norm. That is the case of the position operator $\hat{X}$ and the linear momentum operator $\hat{P}$. In a consistent axiomatic frame, all the eigenfunctions of operators associated to physical properties should belong to a common space. The Hilbert space $\mathcal{H}$ contains only normed vectors. It is then necessary to introduce an extension: the equipped Hilbert space $\mathcal{H}_{e}$. This is not really a space, but a 3-ple, given by

$$
\mathcal{H}_{e}=<\mathcal{S}, \mathcal{H}, \mathcal{S}^{\prime}>
$$

where $\mathcal{S}$ is a nuclear countable Hilbert space Gel'fand, 1967 (i.e. a space of well-behaved functions), $\mathcal{H}$ is the ordinary Hilbert space, and $\mathcal{S}^{\prime}$ is a space isomorphic to the dual of $\mathcal{S}$ (the distributions, such as Dirac's delta, are in $\mathcal{S}^{\prime}$ ). These three spaces satisfy

$$
\mathcal{S} \subset \mathcal{H} \subset \mathcal{S}^{\prime}
$$

The following theorem, due to Gel'fand [Gel'fand, 1967], that we reproduce without proof, states the necessary conditions to operate on $\mathcal{S}^{\prime}$ in the usual way:

"Let $\mathcal{H}_{e}=<\mathcal{S}, \mathcal{H}, \mathcal{S}^{\prime}>$ be an equipped Hilbert space. If the symmetric and linear operator $\hat{A}$ acting on the space $\mathcal{S}$ admits a self-adjoint extension $\overline{\mathbf{A}}$ on $\mathcal{H}$, then $\overline{\mathbf{A}}$ admits a complete system of eigendistributions $\left\{e_{r}\right\}$ in $\mathcal{S}^{\prime}$ with real eigenvalues".

We now define the action of the operators $\hat{X}$ and $\hat{P}$ on $\mathcal{S}$ (in the corresponding representation) in the following way:

$$
\begin{aligned}
\hat{X} \phi_{r}(x) & =x \phi_{r}(x) \\
\hat{P} \phi_{r}(p) & =p \phi_{r}(p)
\end{aligned}
$$

where $\left\{\phi_{r}\right\}$ is a complete set [2] The extension of the operators (required by the theorem) can be achieved following Gel'fand and Shilov Gel'fand, 1967.

Gel'fand's theorem then legalizes the use of eigenfunctions of infinite norm within the formal structure of the theory.

\section{AXIOMATICS}

Every axiomatic formulation must explicit its background (i.e. the set of all presuppositions of the theory), its basis of primitive concepts (i.e. the set of non-definite concepts that define the derived concepts according to the building rules explicited in the background), its axioms, and its conventions.

There are three kinds of axioms in a theory: formal axioms, physical axioms, and semantical axioms. The formal axioms are of a purely mathematical type and they refer only to conceptual objects. The physical axioms represent objective physical laws. The semantical axioms establish the relations among signs, physical objects and properties of physical objects; in this way they characterize the meaning of the primitives and they set the reference class of the theory.

We next give the background of our formulation of nonrelativistic quantum mechanics for one microsystem $\left(\mathrm{T}_{Q M}\right)$. 


\subsection{FORMAL BACKGROUND}

$\mathbf{P}_{\mathbf{1}}$ Two-valued ordinary logic

$\mathbf{P}_{2}$ Formal semantics [Bunge, 1974a] Bunge, 1974b]

$\mathbf{P}_{\mathbf{3}}$ Mathematical analysis with its presuppositions and generalized functions theory Gel'fand, 1964] Jones, 1966

$\mathbf{P}_{4}$ Probability theory

$\mathbf{P}_{5}$ Group theory

\subsection{MATERIAL BACKGROUND}

$\mathbf{P}_{6}$ Chronology

$\mathbf{P}_{\boldsymbol{7}}$ Euclidean physical geometry Bunge, 1967a

$\mathbf{P}_{\mathbf{8}}$ Physical theory of probability Popper, 1959]

$\mathbf{P}_{9}$ Dimensional analysis

$\mathbf{P}_{10}$ Systems theory

$\mathbf{P}_{11}$ Classical electrodynamics

\subsection{REMARKS}

By chronology we understand the set of theories of time. We adopt here a relational theory for the local time, in which a function is defined such that it maps pairs of events related to a given reference system into a segment of the real line Bunge, 1967a.

The theory of systems deals with physical systems and the relations among them (a physical system is "... anything existing in space-time and such that it either behaves or is handled as a whole in at least one respect" [Bunge, 1967a]). This theory has been axiomatized by Bunge [Bunge, 1967a], and its basis of primitive concepts includes the physical sum or juxtaposition $(\dot{+})$, and the physical product or superposition $(\dot{x})$.

Finally, the inclusion of classical electrodynamics will allow, by means of the axiom $\mathbf{A}_{\mathbf{4 2}}$, the study a microsystem under the influence of an external classical field. The removal of $\mathbf{P}_{\mathbf{1 1}}$ causes the axioms $\mathbf{A}_{\mathbf{3 7}}, \mathbf{A}_{\mathbf{3 8}}$, and $\mathbf{A}_{42}$ to be meaningless.

Let's turn now to the generating basis.

\subsection{GENERATING BASIS}

The conceptual space of the theory is generated by the basis B of primitive concepts, where

$$
\mathrm{B}=\left\{\Sigma, \bar{\Sigma}, \mathrm{E}_{3}, \mathrm{~T}, \mathcal{H}_{e}, \mathcal{P}, \mathrm{A}, \mathrm{G}, \hbar\right\}
$$

The elements of the basis will be semantically interpreted by means of the axiomatic basis of the theory, with the help of some conventions [3]. 


\subsection{DEFINITIONS}

$\mathbf{D}_{1}$ eiv $\hat{A}={ }_{D f}$ eigenvalue of $\hat{A}$

$\mathbf{D}_{\mathbf{2}}[\hat{A}, \hat{B}]={ }_{D f} \hat{A} \hat{B}-\hat{B} \hat{A}$

$\mathbf{D}_{\mathbf{3}} \Psi={ }_{D f}\left\{\alpha \mid \psi_{0}>:(\alpha \in \mathcal{C}\right.$, with $|\alpha|=1) \wedge\left(\mid \psi_{0}>\in \mathcal{H}\right.$ is a fixed vector $\left.)\right\}$ is a ray in $\mathcal{H}$

$\mathbf{D}_{4}$ If $|\psi>\in \Psi \subset \mathcal{H} \Rightarrow| \psi>$ is a representative of $\Psi$

$\mathbf{D}_{5}$ If the spectrum of $\hat{A}$ is continuous $\Rightarrow$

$<\psi|\hat{A}| \phi>={ }_{D f} \int d a d b<\psi|a><a| \hat{A}|b><b| \phi>=\int d a d b \psi^{*}(a) A_{a b} \phi(b)$

$\mathbf{D}_{6}$ If the spectrum of $\hat{A}$ is discrete $\Rightarrow$

$<\psi|\hat{A}| \phi>={ }_{D f} \sum_{i, j}<\psi\left|a_{i}><a_{i}\right| \hat{A}\left|b_{j}><b_{j}\right| \phi>=\sum_{i, j} \psi_{i} A_{i j} \phi_{j}$

$\mathbf{D}_{7} \Psi . \Phi={ }_{D f}|\langle\psi \mid \phi\rangle|$

$\mathbf{D}_{\mathbf{8}} \mathcal{U}={ }_{D f}\left\{\alpha \hat{U}_{0}:(\alpha \in \mathcal{C}\right.$, with $|\alpha|=1) \wedge\left(\hat{U}_{0}\right.$ is a fixed unitary operator on $\left.\left.\mathcal{H}\right)\right\}$ is a ray operator on $\mathcal{H}$

$\mathbf{D}_{\mathbf{9}}$ If $\hat{U} \in \mathcal{U} \Rightarrow \hat{U}$ is a representative of $\mathcal{U}$

$\mathbf{D}_{10}$ If $(|\psi\rangle \in \Psi) \wedge\left(\left|\psi^{\prime}\right\rangle \in \Psi\right) \wedge\left(\left|\psi^{\prime}\right\rangle=e^{i \theta}|\psi\rangle\right) \Rightarrow\left|\psi^{\prime}\right\rangle={ }_{D f}$ gauge transformed by a gauge transformation of the first kind of $|\psi\rangle$.

\subsection{AXIOMATIC BASIS}

$\mathrm{T}_{Q M}$ is a finite-axiomatizable theory, whose axiomatic basis is Bunge, 1974a

$$
\mathcal{B}_{A}\left(T_{Q M}\right)=\bigwedge_{i=1}^{42} \mathbf{A}_{i}
$$

where the index $i$ runs over the axioms.

\subsection{AXIOMS}

\section{GROUP I: SPACE AND TIME}

$\mathbf{A}_{1} \quad \mathrm{E}_{3} \equiv$ tridimensional euclidean space.

$\mathbf{A}_{2} \quad \mathrm{E}_{3} \hat{=}$ physical space.

$\mathbf{A}_{3} \mathrm{~T} \equiv$ interval of the real line $\mathrm{R}$.

$\mathbf{A}_{4} \mathrm{~T} \hat{=}$ time interval.

$\mathbf{A}_{5}$ The relation $\leq$ that orders $\mathrm{T}$ means "before to" $\vee$ "simultaneous with".

\section{GROUP II: MICROSYSTEMS AND STATES}

$\mathbf{A}_{6} \Sigma, \bar{\Sigma}$ : non-empty, denumerable sets.

$\mathbf{A}_{\mathbf{7}} \forall \sigma \in \Sigma, \sigma$ denotes a microsystem. In particular, $\sigma_{0}$ denotes absence of microsystem.

$\mathbf{A}_{\mathbf{8}} \forall \bar{\sigma} \in \bar{\Sigma}, \bar{\sigma}$ denotes environment of some system. In particular, $\bar{\sigma}_{0}$ denotes the empty environment, $<\sigma, \bar{\sigma}_{0}>$ denotes a free microsystem, and $\left\langle\sigma_{0}, \bar{\sigma}_{0}>\right.$ denotes the vacuum.

A $\forall \forall<\sigma, \bar{\sigma}>\in \Sigma \times \bar{\Sigma}, \exists \mathcal{H}_{e} \ni \mathcal{H}_{e}=<\mathcal{S}, \mathcal{H}, \mathcal{S}^{\prime}>\equiv$ equipped Hilbert space.

$\mathbf{A}_{10}$ There exists a one-to-one correspondence between physical states of $\sigma \in \Sigma$ and rays $\Psi \subset \mathcal{H}$. 


\section{GROUP III: OPERATORS AND PHYSICAL QUANTITIES}

$\mathbf{A}_{11} \mathcal{P} \equiv$ non-empty family of functions on $\Sigma$.

$\mathbf{A}_{12} \mathrm{~A} \equiv$ ring of operators on $\mathcal{H}_{e}$.

$\mathbf{A}_{13} \forall \mathcal{A} \in \mathcal{P}, \mathcal{A}$ designates a property of $\sigma \in \Sigma$.

$\mathbf{A}_{14}(\forall \mathcal{A} \in \mathcal{P}) \exists \hat{A} \in \mathrm{A} \ni \hat{A} \hat{=} \mathcal{A}$.

$\mathbf{A}_{15}$ (Hermiticity and linearity)

$(\forall \sigma \in \Sigma) \wedge \ni t_{0}$ is fixed $) \wedge(\forall \hat{A} \in \mathrm{A} \ni \hat{A} \hat{=} \mathcal{A}, \mathcal{A} \in \mathcal{P})$ if $\left|\psi_{1}>,\right| \psi_{2}>\in \mathcal{H}_{e} \Rightarrow$

1. $\hat{A}: \mathcal{H}_{e} \rightarrow \mathcal{H}_{e} \ni \hat{A}\left[\lambda_{1}\left|\psi_{1}>+\lambda_{2}\right| \psi_{2}>\right]=\lambda_{1} \hat{A}\left|\psi_{1}>+\lambda_{2} \hat{A}\right| \psi_{2}>$ with $\lambda_{1}, \lambda_{2} \in \mathcal{C}$

2. $\hat{A}^{\dagger}=\hat{A}$ on $\mathcal{H}$.

A 16 (Probability densities)

$(\forall<\sigma, \bar{\sigma}>\in \Sigma \times \bar{\Sigma}) \wedge(\forall \hat{A} \in \mathrm{A} \ni \hat{A} \hat{=} \mathcal{A}, \mathcal{A} \in \mathcal{P}) \wedge(\forall|a>\in \mathcal{H} \ni \hat{A}| a>=a \mid a>) \wedge(\forall \mid \psi>\in \Psi \subset \mathcal{H}$ that corresponds to the state of $\sigma$ when it is influenced by $\bar{\sigma})$ :

$\langle\psi \mid a\rangle\langle a \mid \psi\rangle \equiv$ probability density for the property $\mathcal{A}$ when $\sigma$ is associated to $\bar{\sigma}$

(i.e. $\int_{a_{1}}^{a_{2}}\langle\psi \mid a\rangle\langle a \mid \psi\rangle d a$ is the probability for $\sigma$ to have an $\mathcal{A}$-value in $\left[a_{1}, a_{2}\right]$ ).

$\mathbf{A}_{17}(\forall \sigma \in \Sigma) \wedge(\forall \bar{\sigma} \in \bar{\Sigma})$ the ray $\Psi$ corresponding to a state of $\sigma$ is the null ray on the border of the accesible region for the system $\sigma \dot{+} \bar{\sigma}$.

A $_{18}(\forall \sigma \in \Sigma) \wedge(\forall \hat{A} \in \mathrm{A}) \wedge(\forall a \ni$ eiv $\hat{A}=a) a$ is the sole value that $\mathcal{A}$ takes on $\sigma$, given that $\hat{A} \hat{=} \mathcal{A}$. (SA)

$\mathbf{A}_{19} \hbar \in \mathcal{R}^{+}$.

$\mathbf{A}_{20}[\hbar]=L M T^{-1}$.

\section{GROUP IV: SYMMETRIES AND GROUP STRUCTURE}

$\mathbf{A}_{21}$ (Unitary operators)

$(\forall<\sigma, \bar{\sigma}>\in \Sigma \times \bar{\Sigma}) \wedge(\forall \hat{A} \in \mathrm{A} \ni \hat{A} \hat{=} \mathcal{A}, \mathcal{A} \in \mathcal{P})$ if $\exists \hat{U} \ni \hat{U}^{\dagger}=\hat{U}^{-1} \Rightarrow \hat{A}^{\prime}=\hat{U}^{\dagger} \hat{A} \hat{U} \hat{=} \mathcal{A}$.

$\mathbf{A}_{22} \forall<\sigma, \bar{\sigma}_{0}>\in \Sigma \times \bar{\Sigma} \exists \hat{D}(\tilde{G})$, unitary ray representation of some central non-trivial extension of the universal covering group $\bar{G}$ of a Lie group $G$ by a one-dimensional abelian group on $\mathcal{H}$.

$\mathbf{A}_{23}$ The Lie algebra $\mathcal{G}$ of the group $G$ is generated by $\left\{\hat{H}, \hat{P}_{i}, \hat{K}_{i}, \hat{J}_{i}\right\} \subset A$.

$\mathbf{A}_{24}$ (Algebra structure)

The structure of $\tilde{\mathcal{G}}$, Lie algebra of $\tilde{G}$ is:

$$
\begin{aligned}
& {\left[\hat{J}_{i}, \hat{J}_{j}\right]=i \hbar \epsilon_{i j k} \hat{J}_{k}} \\
& {\left[\hat{J}_{i}, \hat{K}_{j}\right]=i \hbar \epsilon_{i j k} \hat{K}_{k}} \\
& {\left[\hat{J}_{i}, \hat{P}_{j}\right]=i \hbar \epsilon_{i j k} \hat{P}_{k}} \\
& {\left[\hat{K}_{i}, \hat{H}\right]=i \hbar \hat{P}_{i}} \\
& {\left[\hat{K}_{i}, \hat{P}_{j}\right]=i \hbar \delta_{i j} \hat{M}} \\
& {\left[\hat{J}_{i}, \hat{H}\right]=0 \quad\left[\hat{K}_{i}, \hat{K}_{j}\right]=0 \quad\left[\hat{P}_{i}, \hat{P}_{j}\right]=0 \quad\left[\hat{P}_{j}, \hat{H}\right]=0} \\
& {\left[\hat{J}_{i}, \hat{M}\right]=0 \quad\left[\hat{K}_{i}, \hat{M}\right]=0 \quad\left[\hat{P}_{i}, \hat{M}\right]=0 \quad[\hat{H}, \hat{M}]=0}
\end{aligned}
$$

where $\hat{M}$ is an element of the Lie algebra of a one-parameter subgroup (which is used to extend $\bar{G}$ ). 
$\mathbf{A}_{25} G$ is the Galilei group.

$\mathbf{A}_{26} \hat{H}$ is the time-translations generator.

$\mathbf{A}_{27} \forall<\sigma, \bar{\sigma}>\in \Sigma \times \bar{\Sigma}$, eiv $\hat{H}=E$ represents the energy value of $\sigma$ when it is influenced by $\bar{\sigma}$.

$\mathbf{A}_{28} \hat{P}_{i}$ is the generator of spatial translations on the cartesian coordinate axis $X_{i}$.

$\mathbf{A}_{29} \forall<\sigma, \bar{\sigma}>\in \Sigma \times \bar{\Sigma}$, eiv $\hat{P}_{i}=p_{i}$ represents the $i$-component of the linear momentum of $\sigma$.

$\mathbf{A}_{30} \hat{J}_{i}$ is the generator of spatial rotations around the cartesian coordinate axis $X_{i}$.

$\mathbf{A}_{31} \forall<\sigma, \bar{\sigma}>\in \Sigma \times \bar{\Sigma}$, eiv $\hat{J}_{i}=j_{i}$ represents the $i$-component of the angular momentum of $\sigma$.

$\mathbf{A}_{32} \hat{K}_{i}$ is the generator of pure transformations of Galilei on the axis $X_{i}$.

A $33 \hat{M}$ has a discrete spectrum of real and positive eigenvalues.

$\mathbf{A}_{\mathbf{3 4}} \forall<\sigma, \bar{\sigma}>\in \Sigma \times \bar{\Sigma}$, eiv $\hat{M}=\mu$ represents the mass of $\sigma$.

$\mathbf{A}_{35} \forall<\sigma, \bar{\sigma}>\in \Sigma \times \bar{\Sigma}$, if $\hat{X}_{i}={ }_{D f} \frac{1}{\mu} \hat{K}_{i}$, then eiv $\hat{X}_{i}=x_{i}$ represents the $i$-component of the position of $\sigma$. $(\mathrm{SA})$

\section{GROUP V: GAUGE TRANSFORMATIONS AND ELECTRIC CHARGE}

$\mathbf{A}_{\mathbf{3 6}}(\forall<\sigma, \bar{\sigma}>\in \Sigma \times \bar{\Sigma}) \exists \hat{Q} \in \mathrm{A} \ni(\hat{Q} \neq \hat{I}) \wedge([\hat{Q}, \hat{A}]=0 \forall \hat{A} \in \mathrm{A})$.

$\mathbf{A}_{\mathbf{3 7}} \hat{Q}$ has a discret spectrum of real eigenvalues.

$\mathbf{A}_{\mathbf{3 8}} \hat{Q}$ is the generator of gauge transformations of the first kind.

$\mathbf{A}_{39} \forall<\sigma, \bar{\sigma}>\in \Sigma \times \bar{\Sigma}$, eiv $\hat{Q}=q$ represents the charge of $\sigma$.

$\mathbf{A}_{40}$ There exists one and only one normalized state with eiv $\hat{Q}=0$, named the neutral state.

$\mathbf{A}_{41}$ There exists one and only one normalizable state, named vacuum, that is invariant under $\hat{D}(\tilde{G})$ and under gauge transformations of the first kind.

$\mathbf{A}_{42}$ If $\sigma \in \Sigma$, eiv $\hat{M}=\mu \neq 0$, eiv $\hat{Q}=e$ and $\left\langle A_{0}, \vec{A}\right\rangle$ are the components of an electromagnetic quadripotential that represents the action of $\bar{\sigma} \neq \bar{\sigma}_{0}$ on $\sigma \Rightarrow$

$$
\hat{H}=\frac{1}{2 \mu}\left(\hat{\vec{P}}-\frac{e}{c} \vec{A}\right)^{2}+\frac{e}{c} A_{0}-g_{l} \frac{\hbar e}{m c} \vec{B} \cdot \hat{\vec{\sigma}}
$$

where $\vec{B}$ has the usual meaning that follows from $\mathbf{P}_{\mathbf{1 0}}, \hat{\vec{\sigma}}$ is specified in $\mathbf{T}_{\mathbf{1 3}}$ and $g_{l}$ is the gyromagnetic factor of the microsystem.

\subsection{REMARKS}

From the axioms, it can be seen that the algebra $\mathcal{S}$ of the symmetry group $\mathrm{S}$ of $\mathrm{T}_{Q M}$ for $<\sigma, \bar{\sigma}_{0}>\in \Sigma \times \bar{\Sigma}$ consists of two ideals: an 11-dimensional ideal corresponding to the central extension of the algebra of the universal covering group of the Galilei group by a one-dimensional Lie algebra, and a one-dimensional abelian ideal corresponding to the $\mathrm{U}(1)$ algebra, whose generator is $\hat{Q}$. Stated matematically, $\mathrm{S}=\tilde{G} \otimes \mathrm{U}(1)$.

In the case of $\sigma \neq \bar{\sigma}_{0}$, the group of symmetries will depend on the explicit form of $\hat{H}$, and its algebra will be some subalgebra of $\mathcal{S}$.

The theorems will show that the physics is mainly contained in the commutation relations given in $\mathbf{A}_{\mathbf{2 4}}$. 


\subsection{DEFINITIONS}

$\mathbf{D}_{11}$ Non-degenerated spectrum of an operator $\hat{A} \ni \hat{A}|\phi>=a| \phi>$ (with given boundary conditions) where $\hat{A} \in \mathrm{A}$ and $\mid \phi>\in \Phi \subset \mathcal{H}={ }_{D f}\{a\} \ni(\forall a \in\{a\} \exists \mid \phi>\in\{|\phi>: \hat{A}| \phi>=a \mid \phi>\}) \wedge(\{a\} \cong\{\mid \phi>\})$

$\mathbf{D}_{12}$ Component of $\mid \psi>$ along $\left|\phi_{k}\right\rangle={ }_{D f}<\phi_{k}|\psi\rangle=c_{k}$

$\mathbf{D}_{13}<\hat{A}>={ }_{D f}<\psi|\hat{A}| \psi>$

$\mathbf{D}_{14} \Delta \hat{A}={ }_{D f} \hat{A}-<\hat{A}>$

$\mathbf{D}_{15}(\Delta \hat{A})^{2}={ }_{D f}<(\hat{A}-<\hat{A}>)^{2}>=<\hat{A}^{2}-<\hat{A}>^{2}>$

$\mathbf{D}_{16}\|\psi\|^{2}={ }_{D f}<\psi \mid \psi>$

$\mathbf{D}_{17} \hat{S}_{i}={ }_{D f} \frac{\hbar}{2} \hat{\sigma}_{i}$

$\mathbf{D}_{18} \hat{L}_{i}={ }_{D f} \epsilon_{i j k} \hat{X}_{j} \hat{P}_{k}$

$\mathbf{D}_{19}$ Time evolution operator $=_{D f} \hat{U}\left(t, t_{0}\right) \ni\left(\hat{U}\left(t, t_{0}\right) \hat{U}\left(t, t_{0}\right)^{\dagger}=\hat{I}\right) \wedge\left(\hat{U}\left(t, t^{\prime}\right) \hat{U}\left(t^{\prime}, t_{0}\right)=\hat{U}\left(t, t_{0}\right)\right) \wedge$ $\left(\hat{U}\left(t_{0}, t_{0}\right)=\hat{I}\right) \wedge\left(\hat{A}(t)=\hat{U}\left(t, t_{0}\right)^{\dagger} \hat{A}\left(t_{0}\right) \hat{U}\left(t, t_{0}\right)\right)$

\subsection{THEOREMS}

In this section we give some illustrative theorems that can be deduced from the axioms.

$\mathbf{T}_{\mathbf{1}}$ (Probability amplitudes)

The probability that the property $\mathcal{A}$ represented by a non-degenerate operator $\hat{A}$ of the composed system $\sigma \dot{+} \bar{\sigma}$ in the state $\Psi$ takes a value $a_{k} \in\left\{a_{k_{1}}, a_{k_{2}}\right\}$ is given by

$$
P\left(a_{k}\right)=\sum_{k \in \Delta k}\left|c_{k}\right|^{2}
$$

$$
\Delta k=\left\{k_{1}, k_{2}\right\} \text { where } c_{k}=<\phi_{k} \mid \psi>\text { and } \mid \phi_{k}>\text { is an eigenvector of } \hat{A}
$$

Proof: see [Bunge, 1967a], p. 252.

$\mathbf{T}_{\mathbf{2}}$ Under the same conditions of $\mathbf{T}_{\mathbf{1}}$, the average of $\hat{A}$ is:

$$
<\hat{A}>=\sum_{k}\left|c_{k}\right|^{2} a_{k}
$$

Proof: from $\mathbf{P}_{\mathbf{4}}$ and $\mathbf{T}_{\mathbf{1}}$.

$\mathbf{T}_{\mathbf{3}}(\forall<\sigma, \bar{\sigma}>\in \Sigma \times \bar{\Sigma}) \wedge(\forall \hat{H} \neq \hat{H}(t) \ni \hat{H}$ is the generator of temporal translations $)$ the time evolution operator is:

$$
\hat{U}\left(t, t_{0}\right)=\exp \left\{-\frac{i}{\hbar} \hat{H}\left(t-t_{0}\right)\right\}
$$

Proof: using $\mathbf{A}_{24}$ and $\mathbf{A}_{26}$.

$\mathbf{T}_{\mathbf{4}}$ (Schrödinger equation)

If $\left|\psi>_{t}=\hat{U}\left(t, t_{0}\right)\right| \psi>_{t_{0}} \in \Psi$ is a representative of the state of $\sigma \in \Sigma$ when $\sigma$ is influenced by $\bar{\sigma} \in \bar{\Sigma}$ then $\mid \psi>_{t}$ satisfies:

$$
\hat{H} \mid \psi>_{t}=i \hbar \frac{\partial|\psi\rangle_{t}}{\partial t}
$$

Proof: from $\mathbf{T}_{\mathbf{3}}$. 
$\mathbf{T}_{\mathbf{5}} \forall<\sigma, \bar{\sigma}_{0}>\epsilon \Sigma \times \bar{\Sigma}, \hat{H}=\frac{\hat{P}^{2}}{2 \mu}$

Proof: from $\mathbf{A}_{\mathbf{2 4}}$ (see Hamermesh, 1960).

$\mathbf{T}_{\mathbf{6}} \forall<\sigma, \bar{\sigma}>\in \Sigma \times \bar{\Sigma}$ the properties $\mathcal{A}, \mathcal{B} \in \mathcal{P}$ take definite values at the same time if and only if the associated operators $\hat{A}$ and $\hat{B}$ have the same eigenvectors.

Proof: using $\mathbf{D}_{\mathbf{1 4}}$.

$\mathbf{T}_{\mathbf{7}}$ The operators $\hat{A}$ and $\hat{B}$ of $\mathbf{T}_{\mathbf{6}}$ have a common basis of eigenvectors if and only if they commute.

Proof: using $\mathbf{D}_{\mathbf{5}}$.

$\mathbf{T}_{\mathbf{8}}$ (Heisenberg's inequalities)

$(\forall<\sigma, \bar{\sigma}>\in \Sigma \times \bar{\Sigma}) \wedge(\forall \mid \psi>\in \mathcal{H}) \wedge(\forall\{\hat{A}, \hat{B}, \hat{C}\} \subset \mathrm{A} \ni \hat{A} \hat{=} \mathcal{A}, \hat{B} \hat{=} \mathcal{B}, \hat{C} \hat{=} \mathcal{C}$ with $\{\mathcal{A}, \mathcal{B}, \mathcal{C}\} \subset \mathcal{P})$ if $[\hat{A}, \hat{B}]=i \hat{C} \Rightarrow$

$$
(\Delta \hat{A})^{2}(\Delta \hat{B})^{2} \geq|\hat{C}|^{2} / 4
$$

Proof: using $\mathbf{D}_{\mathbf{1 2}}, \mathbf{D}_{\mathbf{1 4}}$, Schwartz's inequality, and the definition $\hat{F}=\hat{A} \hat{B}+\hat{B} \hat{A}$.

COROLLARY: If $\left[\hat{X}_{i}, \hat{P}_{j}\right]=\hbar \delta_{i j} \hat{I}$ then

$$
\Delta \hat{X}_{i} \Delta \hat{P}_{j} \geq \hbar / 2
$$

$\mathbf{T}_{\mathbf{9}}$ (Heisenberg's equation)

$(\forall<\sigma, \bar{\sigma}>\in \Sigma \times \bar{\Sigma}) \wedge(\forall \hat{A} \in \mathrm{A} \ni \hat{A} \hat{=} \mathcal{A}, \mathcal{A} \in \mathcal{P}):$

$$
\frac{d \hat{A}}{d t}=\frac{i}{\hbar}[\hat{H}, \hat{A}]
$$

Proof: from $\mathbf{D}_{\mathbf{1 8}}$ and $\mathbf{T}_{\mathbf{3}}$.

COROLLARY: if $[\hat{H}, \hat{A}]=0 \Rightarrow \hat{A}$ represents a constant of motion.

$\mathbf{T}_{10}(\forall<\sigma, \bar{\sigma}>\in \Sigma \times \bar{\Sigma}) \wedge(\forall \mid \psi>\in \mathcal{H}) \wedge(\forall \hat{A} \in \mathrm{A} \ni \hat{A} \hat{=} \mathcal{A}$ with $\mathcal{A} \in \mathcal{P}) \wedge(\forall \hat{H} \ni[\hat{H}, \hat{A}]=i \hat{C}):$

$$
\Delta \hat{H} \tau_{A} \geq \frac{\hbar}{2}
$$

with $\tau_{A}=\Delta \hat{A} /|d<\hat{A}>/ d t|$.

Proof: from $\mathbf{D}_{\mathbf{1 2}}, \mathbf{T}_{\mathbf{8}}$ and $\mathbf{T}_{\mathbf{9}}$.

$\mathbf{T}_{\mathbf{1 1}}$ If $\hat{J}_{i}$ is the spatial rotations generator around the axis $x_{i} \Rightarrow$

$\left[\hat{J}^{2}, \hat{J}_{i}\right]=0$.

Proof: using $\mathbf{A}_{24}$.

$\mathbf{T}_{\mathbf{1 2}}$ If $\mid j, m>$ is an eigenstate of $\hat{J}^{2}$ and $\hat{J}_{3}$ then

$$
\begin{gathered}
\hat{J}^{2}\left|j, m>=\hbar^{2} j(j+1)\right| j, m> \\
\hat{J}_{3}|j, m>=\hbar m| j, m>
\end{gathered}
$$

with $-j \leq m \leq-m, j$ half-integer.

Proof: from $\mathbf{A}_{\mathbf{2 4}}$ and $\mathbf{T}_{\mathbf{1 1}}$, using $\hat{J}_{ \pm}=\hat{J}_{1} \pm i \hat{J}_{2}$.

$\mathbf{T}_{\mathbf{1 3}}$ (Spin)

If $j=1 / 2 \Rightarrow \hat{\vec{J}}=\left(\hat{J}_{1}, \hat{J}_{2}, \hat{J}_{3}\right)=\frac{\hbar}{2} \hat{\vec{\sigma}}$, with $\hat{\vec{\sigma}}=\left(\hat{\sigma}_{1}, \hat{\sigma}_{2}, \hat{\sigma}_{3}\right)$, and 


$$
\sigma_{1}=\left(\begin{array}{cc}
0 & 1 \\
1 & 0
\end{array}\right) \quad \sigma_{2}=\left(\begin{array}{cc}
0 & -i \\
i & 0
\end{array}\right) \quad \sigma_{3}=\left(\begin{array}{cc}
1 & 0 \\
0 & -1
\end{array}\right)
$$

Proof: from $\mathbf{D}_{\mathbf{5}}$ and $\mathbf{T}_{\mathbf{1 2}}$, using $\hat{J}_{ \pm}$.

$\mathbf{T}_{14} \forall<\sigma, \bar{\sigma}>\in \Sigma \times \bar{\Sigma}, \hat{\vec{J}}=\hat{\vec{L}}+\hat{\vec{S}}$

Proof: from $\mathbf{D}_{\mathbf{1 7}}, \mathbf{D}_{\mathbf{1 8}}$ and $\mathbf{A}_{\mathbf{2 4}}$.

$\mathbf{T}_{15}$ (Superselection rules)

$\forall<\sigma, \bar{\sigma}>\in \Sigma \times \bar{\Sigma}, \mathcal{H}$ decomposes in mutually orthogonal subspaces whose vectors are eigenvectors of $\hat{M}$. The same is valid for the charge operator $\hat{Q}$.

Proof: from $\mathbf{A}_{\mathbf{2 4}}$ and $\mathbf{A}_{\mathbf{3 6}}$.

\subsection{REMARKS}

The theorem $\mathbf{T}_{\mathbf{5}}$ gives the form of $\hat{H}$ for a free microsystem; its expression is deduced from the symmetry group (i.e. the Galilei group). The time-translations generator $\hat{H}$ characterizes the Schrödinger's equation $\left(\mathbf{T}_{\mathbf{4}}\right)$, which in turn enables us to calculate the vectors corresponding to the physical states of the system. It is clear then that the fundamental physical features of the theory are contained in $\mathbf{A}_{\mathbf{2 4}}$.

The theorem $\mathbf{T}_{\mathbf{1 0}}$ should not be taken as the so-called fourth Heisenberg's inequality: $\Delta E \Delta t \geq \hbar / 2$, which is meaningless in our formulation. In fact, being $t$ a parameter and not an operator, this latter relation is not a logical consequence of $\mathbf{T}_{\mathbf{8}}$. In the expression given in $\mathbf{T}_{\mathbf{1 0}}$ only the characteristic time of the statistical evolution of the operator $\hat{A}$ (i.e. $\tau_{A}$ ) appears.

The superselection rule $\left(\mathbf{T}_{\mathbf{1 5}}\right)$ for the mass operator $\hat{M}$ implies the conservation of the microsystem's mass in the processes that can be described within our axiomatic frame (i.e those non-relativistic processes that reduce to a problem involving a microsystem and its environment). This restriction also holds in Galilean Quantum Field Theories: it forbides certain reactions in which annihilation and creation of particles occur Levy-Leblond, 1967. Note that the superselection rule for the mass is a direct consequence of the imposition of physical representations to the Galilei group. In contrast, the corresponding rule for the charge must be presented in a separated axiom.

\section{SEMANTICAL ASPECTS}

The semantical structure of the theory is determined by the semantical rules expressed in the axioms (SA). This set of axioms fixes the factual interpretation of the mathematical formalism, giving the theory a physical status.

The semantical axioms are of two kinds: denotation rules (like $\mathbf{A}_{\mathbf{7}}$ or $\mathbf{A}_{\mathbf{8}}$ ) that relate symbols and referents in a conventional way, and representation rules (like $\mathbf{A}_{\mathbf{1 4}}$ or $\mathbf{A}_{\mathbf{2 1}}$ ) that set correspondences between functions (or other conceptual objects) and properties of referents. These last rules are not conventional. Moreover, they are hypothesis that can be empirically and theoretically contrasted Bunge, 1974a. This fact permits the discussion of the foundations of the theory, giving to the variety of presented hypothesis, a variety of rival interpretations. However, in most of the interpretations the semantical axioms are not clearly identified from the rest of the axioms. This facilitates the propagation of interpretation mistakes.

A semantical axiom that usually appears in the standard formulation of the theory is the so-called von Neumman's projection postulate:

"If the measurement of a physical observable $\mathcal{A}$ (with associated operator $\hat{A}$ ) on a quantum system in the state $|\psi\rangle$ gives a real value $a_{n}$, then, inmediately after the measure, the system evolves from the state $|n\rangle$, where $\hat{A}\left|n>=a_{n}\right| n>"$.

This postulate interprets the collapse of the wave function as a consequence of the act of measuring the property $\mathcal{A}$. In our formulation of $\mathrm{T}_{Q M}$ this postulate plays no role. Morover, it is in contradiction with the 
rest of the axiomatic core: neither the observer nor the measuring apparatus are present in the background or the generating basis. As a consequence, none of the legitimate statements in the theory can refer to them. Our formulation is objective, realistic and literal. The microsystem-apparatus interaction must be studied by the quantum theory of measurement, and there are reasons Cini, 1983 to think that also in this theory the postulate in question can be eliminated.

If $\mathrm{T}_{Q M}$ does not say anything about observers and measurements, which is the kind of entities whose existence is presupposed by it?. To ask this is to ask for the ontology of the theory. Vaguely, the ontology is the answer, given by a theory, to the question "what is there?". More precisely, we understand the ontology in the following restricted sense: the ontology of the theory is the factual restriction of the set formed by the union of the domains of all the variables related to logical quantifiers that appear in the axiomatic basis of the theory (by factual restriction we understand a restriction of the domain to the subset formed by all the non- conceptual elements) [4]. In the axioms, we quantify on the elements of the generatig basis or on conceptual objects generated by it. All the non-conceptual objects of $\mathcal{B}_{A}$ belong to $\Sigma \bigcup \bar{\Sigma}$. That is why we identify this set with the ontology of $\mathrm{T}_{Q M}$. In our restricted sense, the ontology coincides with the reference class of the theory Bunge, 1974a:

$$
R_{F}\left(T_{Q M}\right)=\bigcup_{i=1}^{42} R_{F}\left(\mathbf{A}_{\mathbf{i}}\right)=\Sigma \cup \bar{\Sigma}
$$

$\mathrm{T}_{Q M}$ refers then only to microsystems and its physical environments.

\section{CONCLUSIONS}

We have presented in this work an axiomatization of nonrelativistic quantum mechanics which displays without ambiguity the logical structure of the theory, in such a way that any proposition is either a postulate or a logical consequence of the postulates. In this form, there is no place for statements unrelated to the primitive concepts of the generating basis. Besides, the semantical structure of the theory has been totally explicited. This avoids possible mistakes in the assignation of meaning to the different symbols. These aspects, joined with the formal advantages mentioned in the introduction, enable us to build an axiomatic picture of QM with a realistic and objective interpretation.

It is widely known that, in the subjective interpretations of QM, the state vector gives a complete characterization of only one microsystem. On the other hand, in the realistic statistical interpretations, the state vector describes an ensemble of microsystems. In the interpretation of the axiomatic basis here presented, a ray in a Hilbert space characterizes a single microsystem in a realistic (nonsubjectivistic) way.

The formal structure developed in the present article is apt to study a single microsystem with spin, with or without an external electromagnetic field, and every problem that can be reduced to a one body problem (e.g. hydrogen-like atoms). This limitation will be removed in a future paper, which will generalize the axiomatic frame in such a way that it will encompass the case of a microsystem with an arbitrary number of components. Particular attention will be payed to the symmetrization postulate and the EPR paradox.

\section{ACKNOWLEDGMENTS}

We are grateful to M. Rocca and P. Sisterna for some helpful discussions, and specially to M. Bunge for valuable comments on the preparation of the manuscript.

\section{References}

[Bargmann, 1954] Bargmann, V.: (1954) Ann. of Math., $\underline{59}, 1$.

[Bunge, 1967a] Bunge, M.: (1967) Foundations of Physics, Springer-Verlag, New York. 
[Bunge, 1967b]

Bunge, M.: (1967) "A ghost-free axiomatization of quantum mechanics", in M. Bunge editor, Quantum Theory and Reality, Springer-Verlag, Berlin-Heidelberg-New York.

[Bunge, 1973] Bunge, M.: (1973) Philosophy of Physics, Dordrecht, D. Reidel Publ. Co.

[Bunge, 1974a] Bunge, M.: (1974) Sense and Reference, D. Reidel Publ. Co., Dordrecht and Boston.

[Bunge, 1974b] Bunge M.: (1974) Interpretation and Truth, D. Reidel Publ. Co., Dordrecht and Boston.

[Cini, 1983] Cini, M.: (1983) Il Nuovo Cimento B, $\underline{73}, 27$.

[Gel'fand, 1967] Gel'Fand, I. M and Shilov, G. E: (1967) Generalized Functions, vol. 3, Academic Press, New York.

[Gel'fand, 1964] Gel'fand, I. M ET AL: (1964-1968) Generalized Functions, vol. 1-4, Academic Press, New York.

[Hamermesh, 1960] Hamermesh, M.: (1960) Ann. Phys., 9 , 518.

[Inönu, 1952] InönU, E. ANd Wigner, E. P.: (1952) Il Nuovo Cimento, $\underline{9}, 705$.

[Jones, 1966] Jones, D. S.: (1966) Generalised Functions, McGraw-Hill, London.

[Lèvy-Leblond, 1963] Lèvy-Leblond, J. M.: (1963) J. Math. Phys., $\underline{4}, 776$.

[Levy-Leblond, 1967] LÉvy-Leblond, J. M.: (1967) Commun. math. Pyhs. $\underline{4}, 157$.

[Popper, 1959] Popper, K. R.: (1959) Brit. J. Phil. Sci., 10, 25.

[Von Neumann, 1955] von Neumann, J.: (1955) Mathematical Foundations of Quantum Mechanics, Princeton University Press.

\section{Notes}

[1] Latin indices can take the values 1, 2, 3 .

[2] It may seem that these definitions are somehow restrictive because they depend on the corresponding representations. However, it is shown in Jones, 1966 that there exists an isomorphism between the $x$ and $p$ representations of $\mathcal{S}$ and $\mathcal{S}^{\prime}$, whereas the isomorphism between the representations of $\mathcal{H}$ can be deduced from Parseval's theorem. The existence of these isomorphisms guarantees independence from any particular representation.

[3] We use an informal notation (with the risk of commiting language abuses) instead of exact logical notation that would obscure the physics of the problem.

Some unusual symbols and their meaning: $\hat{=}(" \ldots$ represents..."), $\ni(" \ldots$ such that..." $), \cong($ "... isomorphic to...").

[4] In a strict sense, it should be understood that a X-logy is a theory of X, for all X, and not just a set. However, Quine and others use this word in a different sense, related to the set of entities accepted by the theory. A more precise definition of this last acception is given here. 